\title{
Physikalische Therapie: Höhen- oder Klimatherapie
}

\author{
Jean-Michel Jeannin
}

Basel, Schweiz

\section{Einleitung}

Die physikalische Therapie umfasst eine breite Palette von therapeutischen, prophylaktischen und rehabilitierenden Behandlungsmethoden. Die Methoden sind zum Teil in der Schulmedizin bestens anerkannt und verankert; andere Methoden werden eher der Komplementärmedizin oder gar der Esoterik zugerechnet. Obwohl die Mittel der Physikalischen Therapie (oder Medizin) aufder Physik beruhen, stammt ihre Bezeichnung vom griechi-

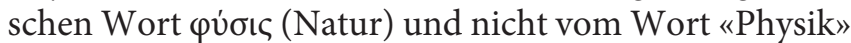
[1]. Die nachfolgende Reihe behandelt das Thema nach dem kennzeichnenden Mittel einer spezifischen physikalischen Therapie gegliedert: Luft, Licht, Wasser, Wärme, Kälte und elektrischer Strom. Die Einteilung ist rein arbiträr, ermöglicht aber die Vielfalt etwas einzufassen. Die einzelnen Themen werden hauptsächlich unter naturwissenschaftlichen Aspekten besprochen. Entsprechend der grossen Bandbreite des Themas muss auf Vollständigkeit verzichtet werden.

\section{Höhen- oder Klimatherapie (Licht)}

\section{Geschichte}

Die physikalische Grundlage einer Höhen- oder Klimatherapie sind die spezifischen klimatischen Bedingungen in höheren Lagen (Abb. 1). Auf 1800 m Meereshöhe ist die Sonneneinstrahlung beispielsweise mehr als doppelt so intensiv wie auf Meereshöhe. Die Höhenluft ist wasser- und staubarm, sodass das Sonnenlicht weniger reflektiert und gestreut wird. Das hauptsächlich wirksame physikalische Element der Höhentherapie ist das UV Licht der Sonne, weshalb bisweilen auch von «Heliothe-

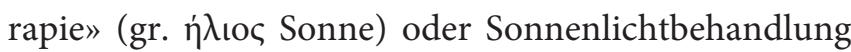
gesprochen wird. Die Ursprünge der Klimatherapie verlieren sich in der Antike. Als Begründer der Sonnenlichtbehandlung in der Neuzeit gilt der Bündner Arzt Oscar
Abb. 1. Spezifische klimatische Bedingungen als Grundlage für die Höhen- oder Klimatherapie.

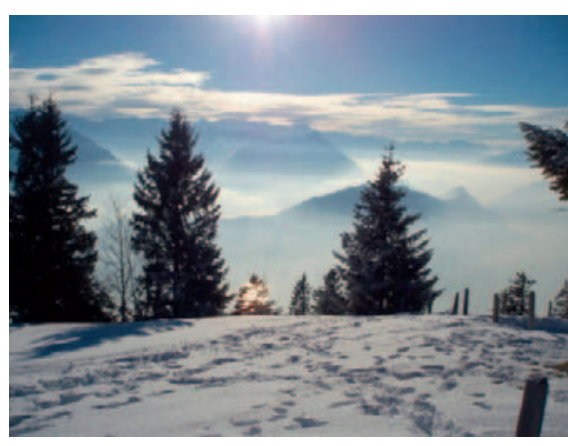

Bernhard (1861-1939) [2]. Er stiess auf das Konzept hierfür in der Trockenfleischherstellung, für die die bakterizide Wirkung des Sonnenlichts genutzt wird. Einen anderen Ansatz verfolgte der aus Mannheim stammende Arzt Alexander Spengler (1827-1901), der die Heilwirkung einer Höhentherapie auf klimatische Faktoren wie verminderten Druck, geringere Luftfeuchtigkeit und niedrigeren Sauerstoffgehalt zurückführte. Seiner Auffassung nach liegt der «Lungenschwindsucht eine uns unbekannte Ernährungsstörung zugrunde» [3]. Die Tuberkulose war Ende des 19. Jahrhunderts zu einem grossen volksgesundheitlichen Problem geworden. Entsprechend dem damaligen Stand des Wissens (aus heutiger Sicht könnte man auch «Unwissen» sagen) war das therapeutische Repertoire eher bescheiden. Mit der Höhentherapie hatte man eine recht gut wirksame Therapie zur Hand, die auch nach der Entdeckung der infektiösen Natur der Tuberkulose verordnet wurde. In allen Einzelheiten wird die Wirkungsweise der Höhentherapie auch heute nicht verstanden [3]. Ende des 18. Jahrhunderts war eine Höhentherapie für weite Teile der Bevölkerung unerschwinglich, weshalb auch Heilstätten für den «minderbemittelten Mittelstand» gegründet wurden. Allein die Anreise z.B. von Norddeutschland nach Davos war eine sehr teure Angelegenheit (siehe «Der Zauberberg» von Thomas Mann).

\section{KARGER}

Fax +497614520714 


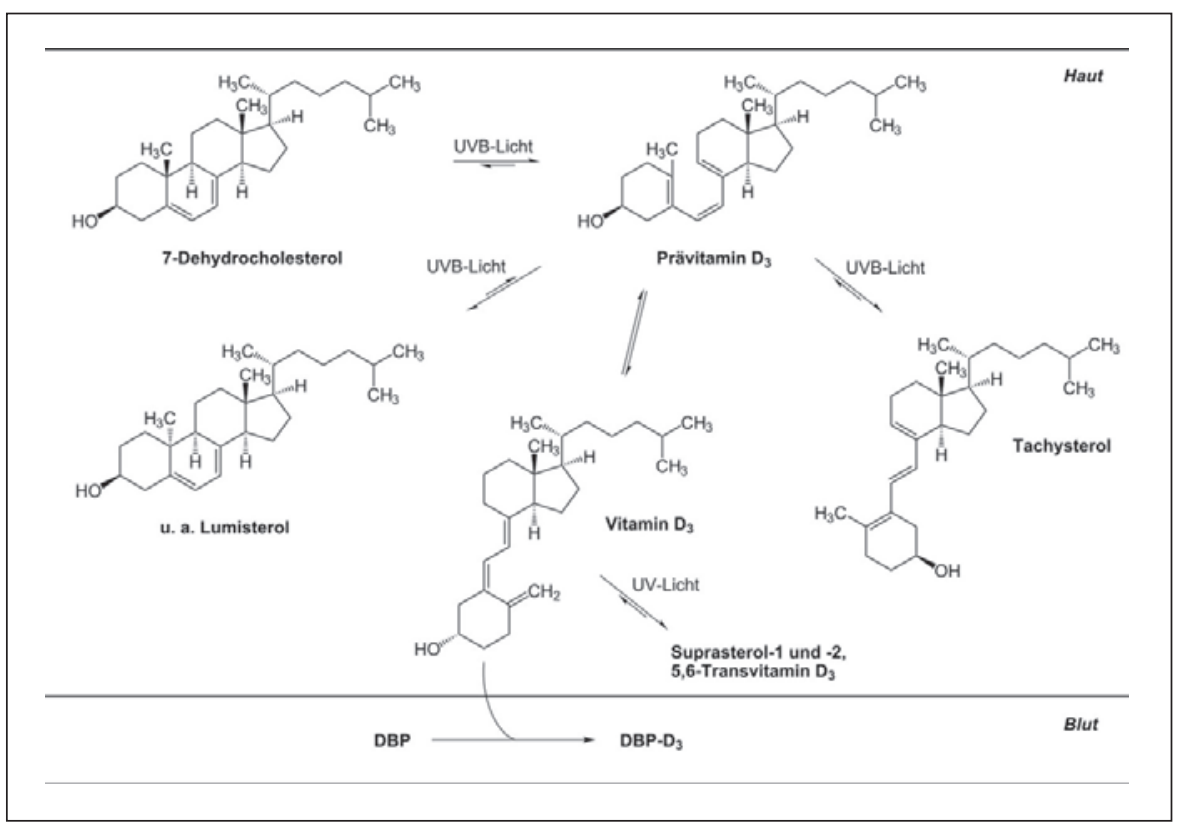

Abb. 2. Vitamin-D-Synthesewege.

\section{Anwendungen und Wirkungsweisen}

Die Anwendung des Sonnenlichts im Rahmen einer höhentherapeutischen Behandlung der Tuberkulose war lange Zeit die einzige bekannte Therapie dieser Krankheit. Nach der Entdeckung der Übertragbarkeit der Tuberkulose nahm man an, das Sonnenlicht würde die Keime direkt töten [4]. Inzwischen weiss man, dass der wichtigste Wirkmechanismus einer Höhentherapie in einer Aktivierung der luminogenen Synthese des Vitamin D3 besteht [4]. Unter UV-Licht der Wellenlängen 290-315 nm («UVB-Licht») bildet der Organismus in der Haut das Vitamin D3 (Cholecalciferol) aus 7-Dehydrocholesterol. Cholecalciferol wird als Prä-Pro-Hormon definiert, das in der Leber durch Hydroxylierung am C-25-Atom in das Pro-Hormon 25-Hydroxyvitamin D metabolisiert wird. In der Niere wird dann nach einer weiteren Hydroxylierung das aktive Vitamin-D-Hormon 1,25-Dihydroxycholecalciferol oder Calcitriol gebildet [5]. Besteht ein Mangel, werden die Makrophagen zu wenig aktiviert, sodass die Synthese von Bakteriziden vermindert ist. Die Höhentherapie der Tuberkulose beruht letztendlich auf einer Anregung der Immunabwehr mittels Steigerung der Vitamin-D-Synthese (Abb. 2).

Nur etwa $10 \%$ des benötigten Vitamin D können über die Nahrung aufgenommen werden, während ca. $90 \%$ in der Haut gebildet werden [6]. Eine ausreichende Lichtexposition ist deshalb entscheidend für eine ausreichende Vitamin-D3-Versorgung. Ein Mangel an Vitamin D löst Störungen der Calciumhomöostase und des Phosphatstoffwechsels aus. Im Kindesalter führt dies zum Krankheitsbild der Rachitis, die mit Deformierungen des Skeletts und Muskelschwäche sowie mit einer erhöhten Infektionsanfälligkeit vergesellschaftet ist. Im Erwachsenenalter kann ein ausgeprägter Vitamin-D-Mangel zu ei- ner Osteomalazie führen. Sie ist durch eine Demineralisierung und einen Umbau der Knochen charakterisiert [5]. Rachitis tritt in Gegenden mit einer verminderten Sonneneinstrahlung (nordische Länder, mit Smog belastete Industriegebiete) und unter Wohnverhältnissen mit wenig Licht gehäuft auf. Bevor eine wirksame Prophylaxe mittels Vitamin-D-Gaben entwickelt wurde, behalf man sich damit, dass z.B. Kindern aus dem Ruhrgebiet ein Aufenthalt in einer Gegend mit intensiverer Sonneneinstrahlung geboten wurde.

Neben den bekannten Funktionen im Calcium- und Phosphatstoffwechsel greift Vitamin D auch in das lokale immunologische Geschehen ein [4]. In der Folge bedeutet ein Vitamin-D-Mangel auch eine Verminderung der Immunabwehr, was sich in einer erhöhten Infektionsanfälligkeit äussert [4]. Eine ausreichende Sonnenlichtexposition ist daher auch für die Aufrechterhaltung einer effizienten Immunabwehr unerlässlich.

\section{Vitamin-D-unabhängige Anwendungen und Wirkungsweisen}

Das UV-Licht der Sonne (Abb. 3) wirkt auch direkt auf das Immunsystem mittels Induktion von entzündungshemmenden Mediatoren und von Substanzen, die die Synthese von entzündungsfördernden Mediatoren unterdrücken. Sonnenlicht moduliert die Expression von oberflächenassoziierten Molekülen, namentlich Adhäsionsmolekülen und Cytokinrezeptoren, und induziert eine Apoptose (programmierter Zelltod) in pathogenetisch relevanten Zellen, d.h. T-Helferzellen. Die Wirkung des UVB-Lichts ist auf die Epidermis beschränkt - im Unterschied zum UVA-Licht, das sowohl auf Zellen der Epidermis als auch auf Zellen der Dermis wirkt [7]. 
Abb. 3. Sonnenlicht als wichtige Ressource.

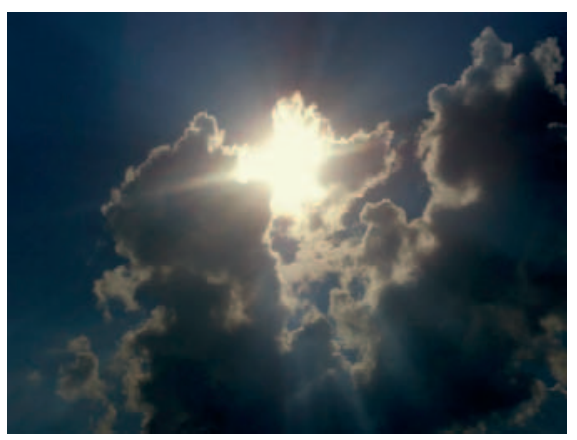

Sonnenlichtexposition erhöht die Energie und die Stimmung. Dazu trägt das Bewusstsein einer Person, gebräunt besser auszusehen, bei. Daneben soll die Exposition die Bildung von $\beta$-Endorphin anregen, und dies in einer Konzentration, die für eine Stimmungsaufhellung und Entspannung ausreicht. Der Mechanismus konnte jedoch nur in einer Studie nachgewiesen werden; in drei anderen Studien gelang der Nachweis nicht. Tatsache ist wiederum, dass Personen, die häufig ein Solarium besuchen, abhängig werden können und dies Aspekte einer Abhängigkeit von einer Substanz aufweisen kann, einschliesslich Entzugserscheinungen [8]. Sonnenbäder vermögen die Schmerzen von Fibromyalgie-Patienten zu reduzieren [8]. Gegenwärtig ist im Detail noch nicht abgeklärt, welche Wirkungen des Sonnenlichts effektiv Vitamin-D-unabhängig sind. Der Stand der Kenntnisse erlaubt auch noch keine präzisen Behandlungsempfehlungen.

\section{Schlussfolgerungen}

Das Leben entwickelt und entfaltet sich gemäss dem Ressourcenangebot. Eine wichtige Ressource ist das Sonnenlicht. Eine ungenügende Exposition ist für viele Krankheiten und Störungen verantwortlich. Das dauernde Fehlen von Sonnenlicht kann zu Katastrophen führen, wie z.B. im Dezember 1952 in London und im Dezember 1962 im Ruhrgebiet geschehen ( Smog-Krise/Katastrophe»). Jenseits von falscher Romantik ist Sonnenlicht existenziell notwendig. Über diese Notwendigkeit hinaus bereitet eine vernünftige Sonnenlichtexposition auch Freude und Vergnügen.

\section{Literatur}

1 Einleitung; in Gutenbrunner C, Glaesener JJ: Rehabilitation, Physikalische Medizin und Naturheilverfahren. Heidelberg, Springer, 2007, p 2.

2 Hofmann H: Begründer der Sonnenlichtbehandlung. Berühmt, verkannt, vergessen. Sprechstunde 2011;4:22-23.

3 Picht E: Ein Jahrhundert Höhentherapie und pathogenetische Theorie der Lungentuberkulose - eine Geschichte der Trugschlüsse? Schweizer Ärztezeitung 2000;81:2507-2510.
4 Aranow C: Vitamin D and the immune system. J Investig Med 2011;59:881-886.

5 Vitamin D (Calciferole); in Deutsche Gesellschaft für Ernährung: Referenzwerte für die Nährstoffzufuhr, ed 1. Frankfurt/Main, Umschau/Braus, 2000.

6 Klimatherapie; in Gutenbrunner C, Glaesener JJ: Rehabilitation, Physikalische Medizin und Naturheilverfahren. Heidelberg, Springer, 2007, pp 90-91.
7 Krutmann J: Therapeutic photoimmunology: photoimmunological mechanisms in photo (chemo)therapy. J Photochem Photobiol B 1998;44:159-164.

8 Juziene A, Moan J: Beneficial effects of UV radiation other than via vitamin $\mathrm{D}$ production. Dermatoendocrinol 2012;4:109-117. 